\title{
Caracterización microbiológica y fisicoquímica de aguas subterráneas de los municipios de La Paz y San Diego, Cesar, Colombia
}

\section{Physicochemical and microbiological characterization of groundwater in the municipalities of La Paz and San Diego, Cesar, Colombia}

\author{
Liliana Vence Márquez', Massiel Rivera González², Yudis Osorio Bayter ${ }^{3}$ \\ y Adriana Beatriz Castillo Sarabia ${ }^{4}$ \\ ${ }^{1}$ Microbióloga Agroindustrial. Especialista tecnológico en Seguridad Industrial. \\ ${ }^{2}$ Microbióloga Agroindustrial. Especialista Técnica en Aplicación de Técnicas \\ Básicas de Biología Molecular. ${ }^{3,4}$ Microbióloga Agroindustrial. \\ ${ }^{1}$ Investigadora Independiente. Valledupar. ${ }^{2}$ Joven Investigador Colciencias. Laboratorio de Nutrición \\ Animal. Corpoica. Bogotá. ${ }^{3}$ nvvestigadora Independiente. ${ }^{4}$ COPETRAN. Valledupar. \\ 1lilyv_1205@hotmail.com, 2mvrivera@corpoica.org.co, \\ 3yudis1931@hotmail.com, ${ }^{4}$ adricastillo-11@hotmail.com,
}

\section{Resumen}

Dentro de las funciones de evaluación, control y seguimiento ambiental de los usos de las fuentes de agua existentes en el Cesar por parte de la Corporación Autónoma Regional del Cesar- CORPOCESAR, se realizó un estudio para evaluar la calidad de aguas subterráneas ubicadas en 93 aljibes en los municipios de La Paz y San Diego (Cesar) en 2009 de acuerdo con lo estipulado en el decreto 1575/07 y la resolución 2115/07 del Ministerio de Protección Social y el Ministerio de Ambiente, Vivienda y Desarrollo Territorial. Se caracterizaron propiedades microbiológicas (determinación de Pseudomona aeruginosa y protozoos patógenos) y fisicoquímicas (conductividad, $\mathrm{pH}$, temperatura, sólidos disueltos totales, salinidad, acidez, alcalinidad, turbidez, cloruros, amonio, nitritos, nitratos, hierro, magnesio, sodio y calcio, estos tres últimos análisis necesarios para calcular la aptitud para riego con el fin de evidenciar la calidad del agua de la que se abastecen los habitantes de estos predios). La detección de P. aeruginosa se realizó mediante filtración por membrana y protozoos mediante la técnica de flotación por centrifugación con sulfato de zinc. De las muestras analizadas en un $84,94 \%$ se encontró $P$. aeruginosa. Se identificaron 5 géneros de protozoos siendo Giardia sp el patógeno con mayor prevalencia, representando un $46,1 \%$, seguido de Criptosporidium sp con un $22,18 \%$; en relación con los resultados fisicoquímicos, estos indicaron que de los aljibes muestreados solo un $4,3 \%$ contienen agua apta para riego sin que su uso acarree riesgos para la salud.

Palabras claves: aljibes, irrigación, protozoos, riesgos.

\section{Abstract}

Among the functions of evaluation, the control and environmental monitoring of the uses of existing water sources in Cesar was studied by the Corporación Autónoma Regional del Cesar, CORPOCE$\mathrm{SAR}$, to evaluate the quality of groundwater located in 93 wells in the municipalities of La Paz and 
San Diego (Cesar) in 2009 in accordance with decree 1575/07 and resolution 2115/07 of the Ministerio de Protección Social (Ministry of Social Protection) and the Ministerio de Ambiente, Vivienda y Desarrollo Territorial (Ministry of Environment, Housing and Territorial Development). Were characterized microbiological properties (determined from Psuedomona aeruginosa and protozoan pathogens) and physiochemical properties (conductivity, $\mathrm{pH}$, temperature, total dissolved solids, salinity, acidity, basicity, turbidity, chlorides, ammonia, nitrites, iron, magnesium, sodium and calcium; the last three elements being necessary for the calculation of the viability of irrigation, with the end of quantifying the quality of the water supplying the inhabitants of these lands). The Detection of $P$. aeruginosa was achieved via filtration through a membrane and protozoa through the flotation technique by centrifugation with zinc sulfate. Of the samples analyzed, $84.94 \%$ contained $P$. aeruginosa. Were identified five genera of protozoa, Giardia sp being the pathogen of highest prevalence at $46.1 \%$, followed by Cryptosporidium sp at $22.18 \%$. Respecting the physiochemical results, only $4.3 \%$ of the wells sampled were determined to be suitable for irrigation without jeopardizing public health and safety.

Key words: wells, irrigation, risk, protozoa.

\section{Introducción}

El agua freática o subterránea es fuente vital para el consumo humano y el uso agrícola; sin embargo, es fácil de agotar porque se renueva muy lentamente (Lanz, 1997). Cuando se contamina es de difícil depuración natural por presentar flujos lentos comparados con el agua superficial de escorrentía, y por su baja presencia de oxígeno que limita la biodegradación. (Herrera y Quintero, 2008). Solo a través de la circulación subterránea, en lo que respecta al paso del agua por las distintas capas terrestres, se tiende a depurar esta agua de partículas y microorganismos patógenos como Pseudomona aeruginosa, Klebsiella $\mathrm{sp}$, Enterococos, coliformes fecales, entre otros (Campos et ál., 2002); que en ocasiones llegan al acuífero por contaminación generada a partir de las actividades humanas como las fosas sépticas, la infiltración de nitratos y otros abonos químicos usados en la agricultura (Fragozo, 2007).

En los municipios de La Paz y San Diego (CesarColombia), se hallan aljibes de aguas subterráneas que son utilizados para el consumo humano, actividades agrícolas y/o ganaderas, pero que se ven afectados por contaminación antrópica (Herrera y Quintero, 2008), estas son aguas que no se les realiza ningún tipo de tratamiento, ni monitoreo medioambiental que incluyan análisis fisicoquímicos y microbiológicos, por lo que se convierten en fuentes de patógenos que causan enfermedades gastrointestinales para la población consumidora.

El objetivo del presente trabajo fue evaluar la calidad microbiológica y fisicoquímica de aguas subterráneas de aljibes ubicados en los municipios de La Paz y San Diego, (Cesar-Colombia) con el fin de determinar la presencia de posibles patógenos que generen enfermedades de origen hídrico, y riesgos sanitarios y comprobar la aptitud para riego del agua proveniente de estos lugares según límites permisibles establecidos en el Decreto 1575/2007 y la Resolución 2115 de 2007 del Ministerio de Ambiente, Vivienda y Desarrollo Territorial, de tal forma que estos estudios sirvan de base para el monitoreo y tratamiento de estas aguas.

\section{Materiales y métodos}

\section{Área de estudio}

La zona de estudio está comprendida por los municipios de La Paz (coordenadas 10²3' N - 73¹0' O) y San Diego (coordenadas $10^{\circ} 20^{\prime} \mathrm{N}-73^{\circ} 10^{\prime} \mathrm{O}$ ), 
Cesar-Colombia. (Figura 1). En estos municipios existen alrededor de 300 aljibes de los cuales se trabajó con una población de 93, distribuidos espacialmente de forma estratégica con el fin de tomar muestras representativas que permitieran hacer una ponderación y, al mismo tiempo, conocer las características de la totalidad de los aljibes ubicados en el área descrita, los cuales fueron ubicados por medio del sistema de posicionamiento global.

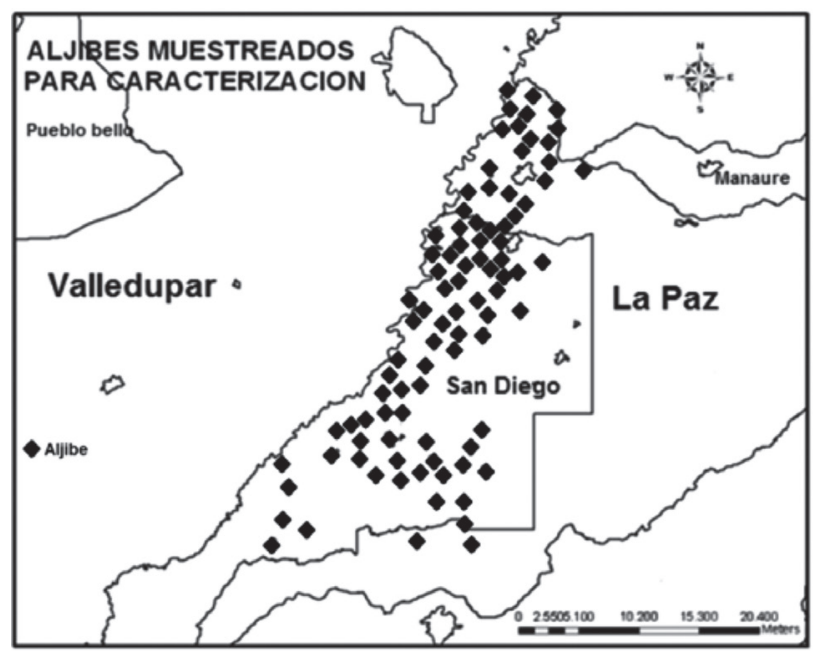

Figura 1. Ubicación geográfica de los aljibes. Fuente: CORPOCESAR, 2011.

\section{Toma de muestras y análisis en laboratorio}

La toma de muestras de agua se realizó acorde con el Decreto 3100 de octubre de 2003 del Ministerio de Protección Social y del Ministerio de Ambiente, Vivienda y Desarrollo Territorial. Para el monitoreo se tomaron muestras puntuales de 93 aljibes. La toma de las muestras se realizó con la ayuda de un tubo plástico capacidad de $1 \mathrm{~L}$, denominado BAILER. Se utilizaron recipientes limpios y estériles para envasar las muestras, 2 réplicas por muestras fueron tenidas en cuenta.

\section{Determinación de la presencia de Pseudomona aeruginosa}

Para la estimación de $P$. aeruginosa se utilizó el método de filtración por membrana (APHA, 2005). El medio de cultivo y equipos utilizados fueron agar Cetrimide ${ }^{\circledR}\left(35^{\circ} \mathrm{C} \pm 2\right)$ y lámpara ultravioleta de onda larga para evidenciar su crecimiento y producción de pigmentos.

\section{Determinación de la presencia de protozoos}

Para la caracterización de protozoos, se utilizó la técnica de Flotación por Centrifugación con Sulfato de Zinc Modificada (APHA, 1997). Estos microorganismos fueron diagnosticados mediante las claves para identificación de Delgado (2001).

\section{Análisis fisicoquímicos de muestras de aguas subterráneas}

El monitoreo de los aljibes contempló los parámetros fisicoquímicos de acidez, alcalinidad, amonio, calcio, cloruros, color aparente y color real, conductividad, dureza cálcica, dureza magnésica, dureza total, hierro, magnesio, nitratos, nitritos, $\mathrm{pH}$, potasio, relación de absorción de sodio (RAS), salinidad, sodio, sólidos disueltos totales, sólidos suspendidos, sólidos totales y sólidos disueltos, temperatura, turbiedad. Se utilizaron botellas plásticas de polietileno, con capacidad para $2 \mathrm{~L}$ de la muestra y fueron preservadas con ácido sulfúrico al 0,02 N. los parámetros para el análisis fisicoquímico se realizaron mediante métodos de titulación y espectrofotométricos, según metodologías establecidas en el Standard Methods (APHA, 2005).

\section{Procesamiento de datos}

En el desarrollo del estudio se realizó un muestreo sistemático y se emplearon los modelos estadísticos de interpolación como son: Ponderación del Inverso de la Distancia IDW (Inverse Distance Weighted), el cual está contenido dentro del Software ArcView $®$ creado por la empresa ESRI, propiedad de CORPOCESAR. Este programa permite la realización de estudios hidrogeológicos que incluyan estudios ambientales, caracterización geología de fuentes de agua, así como para el procesamiento de la información al detallar mediante la generación de mapas de modelamiento geográfico. 


\section{Resultados}

\section{Determinación de Pseudomona aeruginosa}

De las 93 muestras analizadas el 83,87\% presentó $P$. aeruginosa, mayoritariamente en la zona norte y centro de San Diego (ver figura 2). Una de las razones por las cuales se hizo efectiva la presencia de $P$. aeruginosa se debe a las condiciones en la que se encontraban los aljibes en la zona de estudio, referenciando la gran variedad de compuestos orgánicos que utilizan como fuentes de carbono y energía.

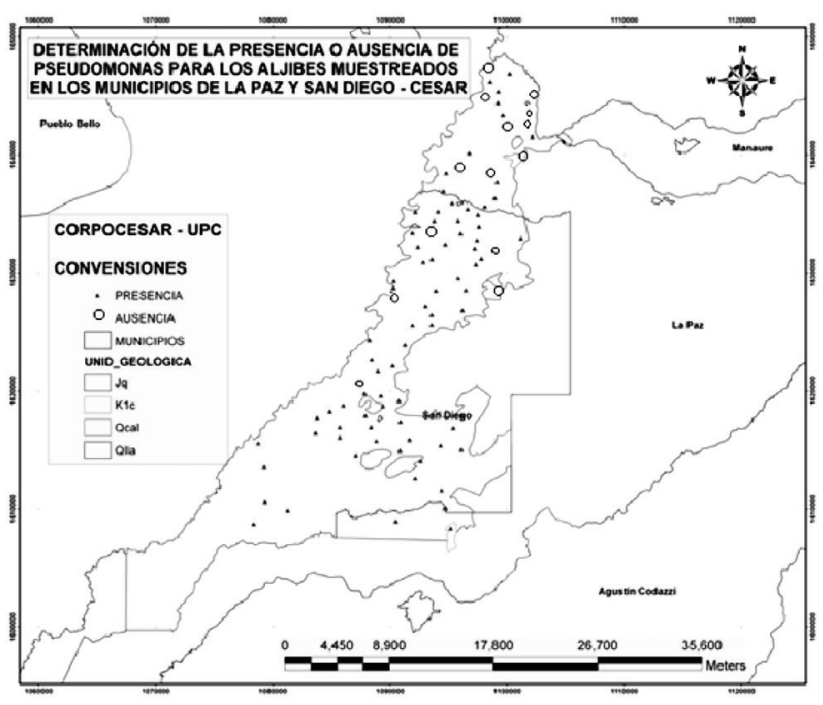

Figura 2. Representación gráfica dada por el software ArcView de los puntos de muestreo de los municipios de La Paz y San Diego, Cesar, respecto con la determinación de la presencia o ausencia de P. aeruginosa. Fuente: propia.

\section{Determinación de protozoos}

De acuerdo con los resultados obtenidos en el análisis para la identificación de protozoos en las 93 muestras, se identificaron los siguientes géneros: Giardia sp $46,1 \%$, Criptosporidium sp $2216 \%$, Entamoeba coli $13,4 \%$, Balantidium coli $8,9 \%$ y Entamoeba histolytica $9,37 \%$ todos en su forma infectante (quistes).

\section{Determinación de análisis fisicoquímicos}

Los resultados obtenidos de los análisis fisicoquímicos realizados a las 93 muestras tomadas, se describen en la Tabla 1, las cuales constan de los valores de los parámetros fisicoquímicos analizados y los valores máximos y mínimos hallados para cada uno de los parámetros analizados.
De acuerdo con el Decreto 1575/2007 y la Resolución 2115/2007 todas las muestras analizadas resultaron ser no aptas para el consumo humano, principalmente desde el punto de vista microbiológico y algunas por sus características fisicoquímicas.

Tabla 1. Valores máximos y mínimos hallados de los parámetros fisicoquímicos evaluados.

\begin{tabular}{lcc}
\hline \multicolumn{1}{c}{ Parametro } & $\begin{array}{c}\text { Valores } \\
\text { minimos }\end{array}$ & $\begin{array}{c}\text { Valores } \\
\text { maximos }\end{array}$ \\
\hline Sólidos suspendidos (SS) & $0,01 \mathrm{mg} / \mathrm{L}$ & $0,45 \mathrm{mg} / \mathrm{L}$ \\
\hline Sólidos totales (ST) & $0,8 \mathrm{mg} / \mathrm{L}$ & $620 \mathrm{mg} / \mathrm{L}$ \\
\hline Sólidos disueltos (SD) & $0,4 \mathrm{mg} / \mathrm{L}$ & $900 \mathrm{mg} / \mathrm{L}$ \\
\hline Dureza total (DT) & $15 \mathrm{mg} / \mathrm{L}$ & $180 \mathrm{mg} / \mathrm{L}$ \\
\hline Dureza cálcica & $10 \mathrm{mg} / \mathrm{L}$ & $105 \mathrm{mg} / \mathrm{L}$ \\
\hline Acidez & $100 \mathrm{mg} / \mathrm{L}$ & $1000 \mathrm{mg} / \mathrm{L}$ \\
\hline Alcalinidad & $2,0 \mathrm{mg} / \mathrm{L}$ & $114 \mathrm{mg} / \mathrm{L}$ \\
\hline Cloruros (Cl) & $0,0035 \mathrm{mg} / \mathrm{L}$ & $0,600 \mathrm{mg} / \mathrm{L}$ \\
\hline Calcio (Ca) & $4 \mathrm{mg} / \mathrm{L}$ & $24 \mathrm{mg} / \mathrm{L}$ \\
\hline Magnesio (Mg) & $0 \mathrm{mg} / \mathrm{L}$ & $33,12 \mathrm{mg} / \mathrm{L}$ \\
\hline Color aparente & $0 \mathrm{UPC}$ & $60 \mathrm{UPC}$ \\
\hline Color real & $0 \mathrm{UPC}$ & $40 \mathrm{UPC}$ \\
\hline Turbidez & $0 \mathrm{UPC}$ & $23,3 \mathrm{UPC}$ \\
\hline Amonio NH4) & $0,493 \mathrm{mg} / \mathrm{L}$ & $23,064 \mathrm{mg} / \mathrm{L}$ \\
\hline Nitratos (NO3) & $0,613 \mathrm{mg} / \mathrm{L}$ & 128,824 \\
\hline Nitritos (NO2) & $0,191 \mathrm{mg} / \mathrm{L}$ & $3,088 \mathrm{mg} / \mathrm{L}$ \\
\hline Hierra(Fe) & $0,011 \mathrm{mg} / \mathrm{L}$ & $0,136 \mathrm{mg} / \mathrm{L}$ \\
\hline Manganeso (Mn) & $2,889 \mathrm{mg} / \mathrm{L}$ & $39,556 \mathrm{mg} / \mathrm{L}$ \\
\hline Sodio (Na) & $1,43 \mathrm{mg} / \mathrm{L}$ & $42 \mathrm{mg} / \mathrm{L}$ \\
\hline Potasio (K) & $0,56 \mathrm{mg} / \mathrm{L}$ & $5,53 \mathrm{mg} / \mathrm{L}$ \\
\hline Temperatura & $27,5{ }^{\circ} \mathrm{C}$ & $31,70{ }^{\circ} \mathrm{C}$ \\
\hline Condinidad & 6,71 & 8,2 \\
\hline
\end{tabular}


Los valores de acidez revelaron que en la mayoría de los puntos se establecen rangos moderados que se hallan distribuidos en grandes extensiones de la zona de estudio, algunos puntos para la zona sur de San Diego mostraron valores muy por encima de lo normal. Los puntos donde se concentraron los valores de mayor acidez, no solo afecta precisamente el punto de muestreo, sino también zonas aledañas, lo que constituye un serio problema para quienes poseen sistemas de tuberías que puedan dañarse por corrosión (Rigola, 1999). Los niveles de alcalinidad evidenciaron marcadas diferencias de la zona norte de San Diego con la occidental de La Paz, lo que demuestra que los más altos valores encontrados se pueden generar debido a la presencia de ácidos orgánicos en el suelo, que contribuyen a que los iones de carbonato y bicarbonato aumenten la alcalinidad, siendo determinante para neutralizar los ácidos (Glynn et al., 2000).

Loa análisis realizados por el software ArcView $®$ mostraron que en la mayoría de los puntos se dan bajas concentraciones de amonio, también se dio para las concentraciones de calcio, el cual se concentra más hacia la zona norte de La Paz y en la parte media de San Diego, bajas o pocas concentraciones de cloruros y de dureza total infiriéndose que el $100 \%$ de los aljibes se encuentra dentro de la norma establecida en los rangos de la Res. 2115 para estos parámetros. El color se encuentra dentro de los niveles aceptables establecidos en la Resolución 2115, la cual establece un rango permisible para consumo humano hasta 15 UPC; el $74,89 \%$ de los aljibes están dentro de la norma y el restante $25,11 \%$ se encontró fuera de norma; lo que puede deberse al contacto del agua con desechos orgánicos en descomposición y están asociados a la presencia de hierro y manganeso (Kiely, 2003).

Para el color real se registraron valores relativamente bajos, lo que quiere decir que el agua en estos puntos no entraba en contacto con desechos orgánicos en descomposición (Kiely, 2003). Datos revelados por el software ArcView indica- ron que el $90,7 \%$ de los aljibes están dentro de la norma establecida, la mayor conductividad se concentra en un punto hacia el sur de San Diego, lo cual indica que en este punto el agua no es apta para irrigación, pues las aguas con valores altos en conductividad pueden afectar la productividad de los cultivos por la alta concentración de sales en las raíces (Romero, 2002). Los valores más altos de dureza cálcica se presentaron al sur de San Diego, mientras que los niveles más elevados de dureza magnésica se encuentran dispersos en la zona de estudio. La presencia de altos valores de dureza cálcica y magnésica se debe al paso del agua subterránea a través de la caliza disolviendo los compuestos de calcio y magnesio (Rigola, 1999).

Valores para el hierro presentaron ciertas fluctuaciones en los puntos muestreados, arrojaron valores altos en algunas zonas, las altas concentraciones de hierro no afectan la salud, pero son preocupantes por razones de estética y gusto, pues produce un sabor metálico al agua, además de contribuir a la acidificación del suelo y a la pérdida de fósforo (Romero, 2002). El 100\% de los aljibes están dentro de los valores establecidos en la Resolución 2115 para los rangos permisibles para magnesio, la presencia de magnesio en los aljibes muestreados puede deberse a las rocas carbonatadas, dolomitas y calizas magnesianas que se encuentran en éstos (Tebbutt, 2001). Resultados para valores de manganeso revelaron altas concentraciones de este metal se encuentran en los puntos pertenecientes a la zona norte de San Diego, el magnesio presentó una coloración negra, lo que indica contaminación por residuos orgánicos como efluentes de silos agrícolas (Tebbutt, 2001); sin embargo, el $100 \%$ de las muestras analizadas se encuentran dentro de los valores establecidos en la Resolución 2115.

El $72,54 \%$ de los aljibes se encontraron fuera de los rangos establecidos en la Resolución 2115 para nitratos, las altas concentraciones de estos indican la disolución de rocas que los contengan o la oxidación de la materia orgánica por acción bacteriana o lixiviados generados a partir de la fertilización 
química que se hace en la región (Kiely, 2003). El $100 \%$ de los aljibes se encuentra fuera de norma establecida para nitritos, las altas concentraciones de estos compuestos en los puntos muestreados indican posible contaminación por sustancias orgánicas (tejidos de animales o vegetales y desechos) (Tebbutt 2001). Aunque se observaron altas concentraciones de $\mathrm{pH}$ dispersos en la zona de estudio, el $100 \%$ de los puntos muestreados se encuentran dentro de la norma.

Las altas concentraciones de sales de nitrato se encontraron con mayor prevalencia al sur de San Diego, lo que genera un problema con los sistemas de regadío, debido a que las altas concentraciones de sales son nocivas para el crecimiento de las plantas y limitan la cantidad de agua que éstas pueden absorber, además incrementan la concentración de elementos tóxicos en el agua (Brassington, 2000). Las bajas concentraciones de sólidos disueltos se encontraron extendidas en los puntos muestreados de la zona norte de La Paz y gran parte de San Diego. Por el contrario, en la zona sur de San Diego se concentraron altos valores encontrados para éste parámetro.

La mayoría de los puntos muestreados (norte de La Paz y San Diego), mostraron las más bajas concentraciones de sólidos suspendidos, y las más altas se encontraron en la zona sureste de San Diego con un $23,5 \%$ del total de las muestras. Las altas concentraciones de sólidos totales disueltos se encontraron en la zona sur de San Diego con un $23 \%$, allí pueden verse afectados muchos cultivos y plantas sensibles (Romero, 2002). Los valores más elevados de turbidez se concentraron en el este de San Diego, alrededor de un $14,88 \%$, lo que indica que se encuentra fuera de los rangos establecidos en la norma.

Los niveles altos de turbidez indican presencia de materiales en suspensión como partículas en suspensión, materia orgánica e inorgánica, organismos planctónicos y microorganismos (Glynn et ál., 2000). Altos niveles de sodio se concentraron en la zona sur de San Diego, las altas concentracio- nes de este elemento son muy perjudiciales para la agricultura, ya que tiende a impermeabilizar los suelos, especialmente en zonas donde el drenaje es deficiente (Romero, 2002). Las altas concentraciones de potasio se observaron esparcidas en diferentes puntos, más especialmente en la zona sur de San Diego; esto puede deberse a que las sales de potasio existentes son difíciles de precipitar en este tipo de aguas además de los aportados por los fertilizantes (Rigola, 1999).

\section{Clasificación de las aguas según el RAS (Norma de Riverside)}

El $47,3 \%$ de las muestras analizadas de agua presentan salinidad media apta para el riego, con contenido medio de sodio y con cierto peligro de acumulación de sodio en el suelo (C2 S2). El 24,7\% (C2 S1), es un agua de salinidad media apta para el riego con bajo contenido de sodio. El $12,9 \%$ es un agua de salinidad alta, puede usarse para riego, siempre y cuando tenga un buen drenaje y posea un contenido medio de sodio con cierto peligro de acumulación (C3 S2). El 5,4\% es un agua de salinidad alta, puede usarse para riego si el suelo tiene buen drenaje, posee un alto contenido de sodio y gran peligro de acumulación en el suelo (C3 S3). El 4,3\% es un agua de baja salinidad, apta para el riego en todos los casos, con bajo contenido de sodio, apta para el riego en la mayoría de los cultivos (C1 S1). El 4,3\% es un agua de salinidad media, apta para el riego con alto contenido de sodio y gran peligro de acumulación en el suelo (C2 S3). El 1,1\% es un agua de salinidad excesiva, no aconsejable para riego, con alto contenido de sodio y gran peligro de acumulación en el suelo (C6 S3) (Bastidas et ál., 2007).

\section{Discusión y conclusiones}

La evaluación realizada de las características microbiológicas y fisicoquímicas de los aljibes ubicados en las zonas rurales de los municipios de La Paz y San Diego, evidenció que en todos los puntos de muestreo los parámetros estudiados 
sobrepasan los rangos normales aptos para que el agua pueda ser utilizada por la población de estos municipios para consumo humano (contacto primario, baño y como alimento). En cambio, se encontró que un 4,3\% del total de los aljibes presentan agua apta para riego, un $76,3 \%$ puede ser utilizada para riego pero su utilización implica ciertos riesgos como aumento en la salinización de los terrenos regados, disminución de la permeabilidad del suelo y disminución de la productividad del cultivo, y un $12,9 \%$ muestran agua no apta para actividades de riego y pastoreo.

La calidad del agua de los aljibes muestreados se encuentra alterada de acuerdo con los resultados descritos en este estudio, además la influencia de las inadecuadas condiciones de higiene del aljibe observadas en la realización de los muestreos como suciedad en el interior, algas, vectores, objetos extraños, transformándose de tal forma en focos infecciosos para la proliferación de microorganismos y representando riesgos potenciales para la salud.

Estudios anteriores, realizados por Gómez y Capachero (2008), quienes evaluaron el nivel de riesgo de contaminación de los pozos de agua subterránea ubicados en el departamento del Cesar utilizando cartografías digitales mediante la modificación del software ArcView, concluyeron que el $32 \%$ del departamento presenta vulnerabilidad alta respecto con el uso de estas fuentes hídricas, situación presentada generalmente en los municipios de Agustín Codazzi, San Diego, La Paz, Valledupar, La Jagua de Ibiríco, Pelaya, Aguachica, Tamalameque y La Gloria (Cesar- Colombia), donde los impactos ambientales ocasionados al recurso hídrico subterráneo por parte de las actividades mineras e infiltración de fluidos contaminados con patógenos o tóxicos son más severos.

La presencia de $P$. aeruginosa en la mayoría de los puntos muestreados, indica contaminación de tipo fecal. Estudios de determinación de P. aeruginosa, en aguas dulces, residuales domésticas y de hospital encontraron que en excretas de dife- rentes animales no fueron halladas, pero si en las excretas humanas (Candela, 2002). Su presencia no solo puede influir en la calidad del agua, sino también que constituye un riesgo para la salud de la población rural que se abastece de esta (Ontiveros, 2009).

Es muy frecuente encontrar este género bacteriano en aguas de aljibes que no han atravesado por un proceso de potabilización, aunque esta bacteria está presente naturalmente en el medio ambiente no es catalogada como patógeno en sentido propio, pero un gran número de estos microorganismos patógenos contenidos en agua que se utiliza para diversas actividades humanas significan un riesgo ya que pueden producir diversas infecciones cutáneas y de las membranas mucosas del ojo, oído, nariz y garganta, enfermedades gastrointestinales entre otras afecciones (De Luca et ál., 2006). La utilización de este microorganismo como indicador de calidad de agua es muy eficiente y debe ser incluida su detección y cuantificación en los análisis de rutina y monitoreo.

Los resultados obtenidos para protozoos indican que en los aljibes muestreados el género Giardia $\mathrm{sp}$ es el protozoo con mayor prevalencia seguido por Criptosporidium sp. Sin embargo, se encontraron complejos formados por la presencia de varios géneros en un mismo aljibe, como fue el caso de un punto de muestreo al occidente de La Paz, que presentó Criptosporidium sp, B. coli, E. coli, y Giardia sp., lo que se convierte en alto riesgo de contaminación y por presentar altos niveles de infección por parte de estos parásitos para quienes se abastecen del recurso hídrico. Las infecciones intestinales por protozoos parásitos constituyen una de las causas más frecuentes de enfermedad entre los seres humanos en el mundo, tres de ellos se destacan como productores de diarrea aguda en pacientes inmunocompetentes, Giardia sp, Cryptosporidium sp, E. histolytica (Lura et ál., 2002).

Los sitios donde se tomaron las muestras, están ubicados en la zona rural de los municipios de La Paz y San Diego, (fincas y haciendas), por lo que 
es inminente la presencia de animales en grandes cantidades y, por ende, la contaminación con microorganismos presentes en el tracto gastrointestinal. Por otra parte, es preciso indicar que muchos de estos puntos de captación presentan fisuras, daños en sus estructuras, tapas mal elaboradas o en mal estado, que permiten el proceso de infección de estas aguas de forma directa o indirecta.

Los resultados de los análisis fisicoquímicos mostraron diferentes problemas asociados a la calidad de agua, entre ellos, problemas de contaminación representados por el elevado contenido de nitratos y nitritos; el exceso en el contenido de sales totales como cloruros, sodio, magnesio, asociados con alta salinidad y conductividad encontrados en algunas zonas, pueden complicar la salud de animales jóvenes y afectar la asimilación de otros nutrientes, además de limitar su uso en la agricultura por parte de los pobladores, en las actividades de riego para cultivos de tubérculos y pastoreo. Resultados similares obtuvieron Fragozo (2007) quien llevó a cabo la ejecución de análisis fisicoquímicos y microbiológicos de 160 muestras de agua subterránea en los municipios de Bosconia, Copey, Valledupar, La Paz y San Diego y la ecorregión del Valle del Río Cesar.

\section{Recomendaciones}

Es importante implementar sistemas de monitoreo, para controlar el grado de contaminación que puedan estar sufriendo los aljibes muestreados debido a la influencia que ejerce las fosas sépticas cercanas y escorrentía de agroquímicos. Es necesario en posteriores estudios ampliar los puntos de muestreo, esto con el fin de conocer como varía la distribución espacio-temporal de la calidad del agua en otros municipios; además realizar estudios de monitoreo con base en las estaciones climatológicas y evaluación de parámetros adicionales como generación de gases tóxicos y determinaciones de algas.

\section{Agradecimientos}

Las autoras agradecen al equipo técnico y administrativo de la Corporación Autónoma Regional del Cesar- CORPOCESAR, a la Universidad del Magdalena y a la Empresa de Servicios Públicos de Valledupar- EMDUPAR S. A. por su apoyo técnico y asesoría científica en la realización de esta investigación

\section{Literatura citada}

1. A.P.H.A, AWWA \& WEF. (2005). Standard Methods for the Examination of Water and Wastewater. $21^{\mathrm{a}} \mathrm{Ed}$ Ed 9222B. Standard Total Coliform Membrane Filter Procedure.

2. Bastidas, M., Moreno, W. \& Duran, T. (2005). Caracterización hidrogeoquímica en el laboratorio de resultados respectivos dentro del marco del proyecto: Evaluación del potencial de aguas subterráneas para riego de los sistemas acuíferos del cono aluvial y la Ilanura aluvial del municipio de Valledupar. Valledupar, Colombia;Convenio CORPOCESAR-UPC.

3. Brassington, R. (2000). Alumbramiento de aguas. Guía para la construcción y mantenimiento de suministros de aguas privadas. Zaragoza: Acribia S. A.

4. Campos, C., Cárdenas, M. \& Guerrero, A. (2002). Comportamiento de los indicadores de contaminación fecal en diferente tipo de agua de la sabana de Bogotá (Colombia). Universidad Javeriana.

5. Candela, L. (2002). Contaminación de las aguas subterráneas: tipo doméstico e industrial. Presente y futuro de las aguas subterráneas en la provincia de Jaén. Recuperado de: http://aguas.igme.es/igme/publica/lib108/ pdf/lib108/in_n7.pdf

6. De Luca L., Zamora A. \& Folabella A. (2006). Bacterias indicadoras de riesgo sanitario aportadas por el riego frente a la supresividad edáfica. Recuperado de: http:// www.prodti.us.es/congreso/carteles/DELUCA.pdf

7. Delgado, A., Polanco, A., Prieto, S., Amich, S. \& Salve, M. (2001) Manual de laboratorio clínico básico. Microbiología. Parasitología Clínica. España: Mac Graw Hill, editores..

8. Fragozo, P. (2007). Evaluación Hidrogeoquímica del agua subterránea en los municipios de Bosconia, Copey, Valledupar, La Paz y San diego ecorregion del valle del Rio Cesar. (Tesis de Maestría en Microbiología). Universidad de Zulia.

9. Glynn, J., Henry, Gary W. \& Heinke. (2000). Ingeniería Ambiental. Aguas subterráneas. México, D. F.: Editorial Prentice Hall. 
10. Gómez, A. \& Capachero, C. (2008). Evaluación de la vulnerabilidad de las aguas subterráneas a la contaminación en el departamento del cesar, aplicando la metodología Drastic. Valledupar, Colombia: CORPOCESAR.

11. Herrera, I. \& Quintero, D. (2008). Microbiología de aguas subterráneas en la región sur del municipio de Valledupar-Cesar. (Trabajo de Grado) Microbiología. Universidad Popular del Cesar.

12. Kiely, G. (2003). Ingeniería Ambiental. Fundamentos, entornos, tecnologías y sistemas de gestión. Madrid: Editorial Mc Graw Hill.

13. Lanz, K. (1997). El libro del agua. Ed. Greenpace España. Madrid, España :Temas de dibale SA.

14. Lura, M., Beltramino, D., Abramovich, B., Carrera, E., Haye, M. \& Contini, L. (2002) El agua subterránea como agente transmisor de protozoos intestinales. Santiago de Chile. Revista chilena de pediatría. 73 (4), 415-424.

15. Ontiveros M. (2009). Agentes patógenos transmitidos por el agua. Disponible en: http://sisbib.unmsm.edu.pe/ bibvirtual/Tesis/Basic/Marchand_P_E/anteced.htm

16. Rigola, L (1999). Tratamiento de aguas industriales, aguas de proceso y residuales. Parámetros de calidad de las aguas. Barcelona, España: Alfaomega Marcombo editores.

17. Romero, J. (2002). Calidad del agua. México D. F: Alfaomega editores.

18. Tebbutt, T. (2001). Fundamentos de control de la calidad del agua. México, D. F: Limusa, editores. 